\title{
Purification and Identification of Bovine Cheese Whey Fatty Acids Exhibiting In Vitro Antifungal Activity
}

\author{
M. Clément, ${ }^{* 1}$ J. Tremblay, ${ }^{*} M$. Lange,$t^{2}$ J. Thibodeau, ${ }^{*}$ and P. Belhumeur ${ }^{\star}$ \\ *Département de microbiologie et immunologie, Université de Montréal, C.P. 6128, succ. Centre-ville, Montréal, Québec, H3C 3J7, Canada \\ †Saputo Inc., 6869 Métropolitain Est St-Léonard, Montreal, Québec, H1P 1X8, Canada
}

\begin{abstract}
Milk lipids contain several bioactive factors exhibiting antimicrobial activity against bacteria, viruses, and fungi. In the present study, we demonstrate that free fatty acids (FFA) derived from the saponification of bovine whey cream lipids are active in vitro at inhibiting the germination of Candida albicans, a morphological transition associated with pathogenicity. This activity was found to be significantly increased when bovine FFA were enriched in non-straight-chain FFA. At low cell density, this non-straight-chain FFA-enriched fraction was also found to inhibit in a dose-dependant manner the growth of both developmental forms of C. albicans as well as the growth of Aspergillus fumigatus. Using an assay-guided fractionation, the main components responsible for these activities were isolated. On the basis of mass spectroscopic and gas chromatographic analysis, antifungal compounds were identified as capric acid (C10:0), lauroleic acid (C12:1), 11-methyldodecanoic acid (iso-C13:0), myristoleic acid (C14:1n-5), and $\gamma$-linolenic acid (C18:3n-6). The most potent compound was $\gamma$-linolenic acid, with minimal inhibitory concentration values of $5.4 \mathrm{mg} / \mathrm{L}$ for C. albicans and $1.3 \mathrm{mg} / \mathrm{L}$ for $A$. fumigatus, in standardized conditions. The results of this study indicate that bovine whey contains bioactive fatty acids exhibiting antifungal activity in vitro against 2 important human fungal pathogens.
\end{abstract}

Key words: whey, free fatty acid, Candida, Aspergillus, antifungal

\section{INTRODUCTION}

Bovine whey was traditionally defined as a natural by-product of the cheese and curd manufacturing industry with low or no commercial interest. However, the

Received October 26, 2007

Accepted March 26, 2008.

${ }^{1}$ Corresponding author: martin.clement@umontreal.ca

${ }^{2}$ Current address: Agropur, 995 Johnson Est, St-Hyacinthe (Quebec), Canada J2S 7V6. imposition of strict controls on its disposal and recent advances in dairy food processing technologies have led to the industrial production of whey derivatives with important nutritional and functional properties (Walzem et al., 2002). More recently, research on whey's benefits has led to the discovery of nonessential trophic factors in bovine whey that can promote health, prevent disease, or both (Shah, 2000; Walzem et al., 2002; Severin and Wenshui, 2005; Huth et al., 2006). Therefore, bovine milk/whey is now recognized as a functional food with interesting properties.

The benefit of bovine milk/whey in preventing infections has long been recognized and has been attributed mainly to immunoglobulins, lysozyme, lactoferrin, lactoperoxidase, and peptides (Severin and Wenshui, 2005). However, bovine milk/whey also contains significant amounts of nonproteic bioactive components, such as sphingolipids and fatty acids, which regulate diverse biological functions, even at low concentrations (Molkentin, 1999; Jensen, 2002). Antimicrobial activity has also been associated with such factors. Indeed, in vitro digestion products of whey-derived sphingolipids and triglycerides enriched in capric and lauric acids exhibit bactericidal effects and thus may protect against food-borne gastroenteritis (Sprong et al., 2001). In addition, some FFA and monoacylglycerides present in abundance in bovine milk/whey display antimicrobial activity against several pathogenic microorganisms including bacteria, viruses, and fungi (Kabara et al., 1972; Isaacs, 2001).

Over the last few decades, invasive fungal infections (IFI) have become a considerable public health problem increasing morbidity and mortality rates in people with AIDS and other immunocompromised individuals (Clark and Hajjeh, 2002; Kullberg and Oude Lashof, 2002). Recent epidemiological surveys indicate that the most common clinical presentations of IFI are due to Candida species, with C. albicans accounting for the majority of cases (Al Jasser and Elkhizzi, 2004; Hajjeh et al., 2004; Tortorano et al., 2004). However, non-albicans Candida species, as well as rare fungal etiologic agents such as Fusarium spp., Paecilomyces lilacinus, and Pseudallescheria boydii, have also been implicated 
(Cimon et al., 2000; Groll and Walsh, 2001; Idigoras et al., 2001; Redding, 2001). In addition, cases of invasive aspergillosis, most commonly due to Aspergillus fumigatus, have reached alarming proportions during the last few decades, accounting in recent years for the majority of reported IFI in some institutions (Denning, 1998; Latge, 1999). These epidemiological data indicate that the spectrum of pathogenic fungi causing IFI in humans is changing over time. Despite extensive use of antifungal therapies, the treatment of IFI remains elusive because some antifungal agents cause toxicity, exhibit poor absorption, and may interact with other drugs (Patterson, 2001; Kontoyiannis and Lewis, 2002). In addition, an increasing number of antifungal agents are becoming less effective because of the emergence of resistant strains of pathogenic fungi (Kontoyiannis and Lewis, 2002; Sanglard and Odds, 2002). Although recent studies have introduced newer promising antifungal agents and alternative immuno/combination therapies, there is a need for new and nontoxic antifungal agents with a wide spectrum of action, especially because emerging pathogenic fungi seem to be particularly resistant to current antifungal agents (Kontoyiannis and Lewis, 2002).

The FFA derived from bovine whey cream were previously shown to interfere in vitro with the germination of C. albicans (Clément et al., 2007), a morphological transition associated with pathogenicity (Lo et al., 1997; Calderone and Fonzi, 2001; Saville et al., 2006; Jackson et al., 2007). In the present study, we demonstrate that FFA derived from the saponification of bovine whey cream lipids are active at inhibiting the germination of C. albicans in vitro. This activity was found to be significantly increased when bovine FFA were enriched in non-straight-chain FFA. In addition, we found that this same fraction could inhibit the growth of $C$. albicans and A. fumigatus, at low cell density. Using an assay-guided HPLC fractionation, the main components responsible for the activity of the nonstraight-chain FFA enriched fraction were identified as capric, lauroleic, 11-methyldodecanoic, myristoleic, and $\gamma$-linolenic acids.

\section{MATERIALS AND METHODS}

\section{Materials and Reagents}

Fresh and unpasteurized bovine whey cream used in this study was from Saputo Inc. (Montreal, Canada). All organic solvents and other material were of the highest purity or HPLC grade (Fisher Scientific, Ottawa, Canada). Capric, 11-methyldodecanoic, myristoleic, and $\gamma$-linolenic acids standards were purchased from Sigma-Aldrich (Oakville, Canada).

\section{Purification of FFA}

Total lipids of fresh and unpasteurized bovine whey cream were extracted according to the Bligh-Dyer procedure (Bligh and Dyer, 1959). Total lipids (10 g) were then subjected to saponification for $60 \mathrm{~min}$ at $60^{\circ} \mathrm{C}$ in a 1-L glass beaker containing $760 \mathrm{~mL}$ of ethanol (96\%) and $16 \mathrm{~g}$ of potassium hydroxide. After cooling down to room temperature, the mixture was filtered $(40 \mu \mathrm{m})$, acidified to $\mathrm{pH} 1$ with $\mathrm{HCl}$, and filtered again. The volume was adjusted to $1 \mathrm{~L}$ with water, and FFA were extracted with hexane $(4 \times 400 \mathrm{~mL})$. The extract $(4 \times$ $400 \mathrm{~mL}$ ) was neutralized by washing with water and dried under nitrogen. The FFA were further purified by solid phase extraction using an aminopropyl disposable column as described by Kaluzny et al. (1985) and Vaghela and Kilara (1995). Essentially, a Mega Bond Elut Flash (25 g) disposable aminopropyl column (Varian, Mississauga, Canada)) was placed onto an Erlenmeyer flask connected to a chemical duty pump (Model WP6111560, Millipore). A vacuum of 10 to $12 \mathrm{kPa}$ was constantly maintained during the procedure. The FFA dissolved in $20 \mathrm{~mL}$ of chloroform were applied under vacuum to the column, which had been prewashed twice with $100-\mathrm{mL}$ portions of hexanes. After eluting nonsaponifiable material with $225 \mathrm{~mL}$ of chloroform/2-propa-

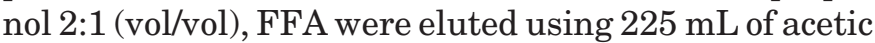
acid/diethyl ether 1:50 (vol/vol). The FFA eluate was dried under nitrogen, and the yield was determined gravimetrically. The resulting FFA were reconstituted to $20 \mathrm{~g} / \mathrm{L}$ in $96 \%$ ethanol and assayed for activity.

\section{Urea Fractionation}

The FFA were fractionated into straight-chain (i.e., saturated) and non-straight-chain (i.e., unsaturated and branched-chain) FFA-enriched fractions by the urea inclusion procedure (Traitler et al., 1988). Essentially, methanol $(12 \mathrm{~mL})$ and urea $(4 \mathrm{~g})$ were added to dried FFA extract $(1 \mathrm{~g})$ placed into a small screw cap vial $(20 \mathrm{~mL})$. Under constant stirring, the mixture was heated to $75^{\circ} \mathrm{C}$, or until it became clear, and then cooled slowly to $2^{\circ} \mathrm{C}$. After completion of urea crystallization at $2^{\circ} \mathrm{C}(15 \mathrm{~h})$, the non-straight-chain FFA present in the methanol phase were separated from the urea crystals by centrifugation $(5 \mathrm{~min})$ at $1,000 \times g$. The nonstraight-chain FFA, as well as the straight-chain FFA present in the urea crystal, were recovered as described by Traitler et al. (1988). Yields were determined gravimetrically. From this procedure, about $25 \mathrm{mg}$ of nonstraight-chain FFA and $900 \mathrm{mg}$ of straight-chain FFA were obtained from approximately $1 \mathrm{~g}$ of FFA. All of the FFA fractions were reconstituted to $20 \mathrm{~g} / \mathrm{L}$ in $96 \%$ ethanol, and binary ethanol dilutions were tested in quadruplicate for antifungal activity. 


\section{HPLC Fractionation}

The non-straight-chain FFA enriched fraction was further separated by reverse-phase HPLC on a semipreparative C18 column (Prep Nova-Pak HR C18, 6 $\mu \mathrm{m}, 60 \AA, 7.8 \times 300 \mathrm{~mm}$, Waters) using a BeckmanCoulter-HPLC Gold system. Non-straight-chain FFA (about $4.5 \mathrm{mg}$ ) dissolved in 50\% ethanol were applied to the column pre-equilibrated with $50 \%$ acetonitrile: $0.1 \%$ TFA, and eluted by a linear gradient to $100 \%$ acetonitrile: $0.1 \%$ TFA from 0 to $70 \mathrm{~min}$ at a flow rate of $8 \mathrm{~mL} / \mathrm{min}$. Ultraviolet detection was used to monitor the effluent at $215 \mathrm{~nm}$. Water $(10 \mathrm{~mL})$ was added to each of the 45 collected HPLC fractions, and they were then extracted 3 times with hexane $(3 \times 10 \mathrm{~mL})$. After drying under nitrogen, each fraction was reconstituted in $30 \mu \mathrm{l}$ of ethanol (70\%), and each was tested in duplicate for antifungal activity. Antifungal assays using commercial FFA were conducted in quadruplicate.

\section{Esterification of FFA}

The FFA were dissolved in $1 \mathrm{~mL}$ of $0.5 \mathrm{~N}$ methanolic$\mathrm{HCl}$ (Supelco, Oakville, Canada) and heated at $50^{\circ} \mathrm{C}$ for 10 min with occasional hand shaking. After cooling to room temperature, water $(1 \mathrm{~mL})$ was added and the resulting fatty acid methyl esters (FAME) were extracted twice with $n$-pentane $(2 \times 1 \mathrm{~mL})$. Organic phases were pooled and evaporated to dryness under nitrogen. The FAME were dissolved in methylene chloride $(0.02$ $\mathrm{mL}$ ) and transferred into small vials with glass inserts (Agilent Technology, Palo Alto, CA). The FAME were kept away from light at $-20^{\circ} \mathrm{C}$ until analysis by gas chromatography.

\section{Gas Chromatography}

Gas chromatographic analysis was performed with a GC-FID 6809N Network System equipped with an Agilent 7683 Series Injector and a flame-ionization detector (Agilent Technology). The FFA were separated on a $30 \mathrm{~m} \times 0.32 \mathrm{~mm}$ ID capillary column coated with a $0.20-\mu \mathrm{m}$ film of polyalkylene glycol (SPB-PUFA, Supelco). After holding the oven temperature at $50^{\circ} \mathrm{C}$ for $2 \mathrm{~min}$, the column was temperature-programmed at $4^{\circ} \mathrm{C} / \mathrm{min}$ to $210^{\circ} \mathrm{C}$. Helium was used as carrier at a velocity of $34 \mathrm{~cm} / \mathrm{s}$. Individual FFA species were identified by comparing retention times to those of known standards (i.e., 37 components FAME mix from Supelco or individual FFA derivatized as FAME).

\section{Mass Spectroscopic Analysis}

Mass spectroscopic analysis was carried out in negative mode using a Micromass Quattro II triple quadru- pole mass spectrometer (Micromass, Manchester, UK) equipped with an electrospray source. Samples dissolved in 50\% isopropanol containing $25 \mathrm{mM}$ triethylamine were infused at a flow rate of $120 \mu \mathrm{L} / \mathrm{h}$. Data were accumulated in MCA mode for $1 \mathrm{~min}$, and analyses were carried out using MassLynx version 3.5 software (Micromass, Waters, MA). Nitrogen was used as curtain gas $(400 \mathrm{~L} / \mathrm{h})$ and nebulizing gas $(20 \mathrm{~L} / \mathrm{h})$. The ESI capillary was set at $2.5 \mathrm{kV}$, whereas the MS analysis was carried out at a cone voltage of $25 \mathrm{~V}$, a scan rate of $300 \mathrm{Da} / \mathrm{s}$ with an interscan delay of $0.1 \mathrm{~s}$ and a scan range of 135 to $500 \mathrm{Da}$. The resolving power was set to obtain unit resolution.

\section{Germination Inhibition Assay}

Candida albicans strain ZK3379 (CAI-4 HWP1-lacZ; Hogan et al., 2004) was used for $\beta$-galactosidase and 2,3-Bis (2-methoxy-4-nitro-5-sulfophenyl)-2Htetrazolium-5-carboxanilide inner salt (XTT) assays. Cells from this strain were grown in YPD (1\% yeast extract, $2 \%$ peptone, $2 \%$ dextrose) at $30^{\circ} \mathrm{C}$ to a density of about $1 \times 10^{8}$ cells $/ \mathrm{mL}$ (hemacytometer). These cells were then washed twice with water and kept on ice until use. Germination was induced by incubating cells (final density of $5 \times 10^{5}$ cells $/ \mathrm{mL}$ ) at $37^{\circ} \mathrm{C}$ in $2.5 \mathrm{~mL}$ of dextrose-free Sabouraud (Joshi et al., 1973), Lee's (Lee et al., 1975), or Spider (Liu et al., 1994) medium, supplemented with FFA. All FFA were dissolved in ethanol. Assays were performed in duplicate in 24-well microtiter plates (Costar 3526, Corning, NY) without agitation. After incubation $(4 \mathrm{~h})$, microtiter plates were centrifuged for $5 \mathrm{~min}$ at $500 \times g$, and supernatants were discarded. The XTT assays were performed immediately and essentially as described previously (Honraet et al., 2005). For $\beta$-galactosidase assays, microtiter plates were frozen at $-80^{\circ} \mathrm{C}$ and later processed as described previously (Kippert, 1995; Hogan et al., 2004) but directly in microtiter plates. Absorbances were measured in 96-well microtiter plates using a microtiter plate reader (Microplate Reader MR600, Dynatech, Alexandria, VA), equipped with filters of $410 \mathrm{~nm}$ ( $\beta$-galactosidase) and $490 \mathrm{~nm}$ (XTT).

\section{Growth Inhibition Assay}

Growth inhibition activity was evaluated in 96-well microtiter plates (Costar 3595) using Sabouraud (A. fumigatus) or dextrose-free Sabouraud (C. albicans) media. Assays were done using spores of $A$. fumigatus or blastospores of $C$. albicans. Spores of A. fumigatus (Jeanine Joly, Université de Montréal) were grown at $25^{\circ} \mathrm{C}$ on slant agar Sabouraud medium. After $7 \mathrm{~d}$ of incubation, spores were harvested by vigorously wash- 
ing slant cultures with $5 \mathrm{~mL}$ of $0.9 \% \mathrm{NaCl}$. Coarse debris were removed by filtering the Aspergillus spore suspension through a sterile cotton plug inserted into a Pasteur pipet. Monodisperse spore suspensions were obtained following brief sonication in a water bath sonicator (Branson 1210, Bransonic, Danbury, CT). Spores of A. fumigatus were adjusted to a density of $5 \times 10^{3} / \mathrm{mL}$ in Sabouraud liquid medium using a hemacytometer. Blastospores of C. albicans were obtained from a single colony of the SC5314 strain (Fonzi and Irwin, 1993) after $24 \mathrm{~h}$ incubation at $30^{\circ} \mathrm{C}$ on solid YPD medium. Blastospores were adjusted to a density of $5 \times 10^{3} / \mathrm{mL}$ in dextrose-free Sabouraud liquid medium using a hemacytometer. Microtiter wells, containing $0.1 \mathrm{~mL}$ of incubating media supplemented with different concentrations of FFA, were inoculated with $0.1 \mathrm{~mL}$ of $A$. fumigatus or $C$. albicans spore suspensions. Microplates were incubated at $37^{\circ} \mathrm{C}$ in atmospheric incubators for $48 \mathrm{~h}$. The minimal inhibitory concentration $\left(\mathbf{M I C}_{50}\right)$ was defined as the lowest concentration reducing by $50 \%$ the optical density at $590 \mathrm{~nm}$ (Microplate Reader MR600, Dynatech) of samples compared with sample-free control (i.e., $1 \%$ ethanol). Antifungal assays were also done as above but in RPMI-1640 buffered with 3-(N-morpholino) propanesulfonic acid (MOPS) at pH 7 as recommended by the National Committee for Clinical Laboratory Standards (see Jacob and Walker, 2005).

\section{RESULTS AND DISCUSSION}

\section{Antifungal Activity of FFA from Bovine Whey Cream Lipids}

Free fatty acids derived from bovine whey cream lipids were previously shown to interfere with the germination of C. albicans in vitro (Clément et al., 2007). Because most bovine milk/whey fatty acids are not free, but rather attached via ester bonds to other molecules (Vaghela and Kilara, 1995), it is difficult to purify large amounts of bovine milk/whey FFA required for fractionation. Therefore, esterified fatty acids were transformed to their free form by saponification of total whey cream lipids, and the resulting FFA were then purified by solid phase extraction using aminopropryl columns. These saponified FFA were assayed for their ability to inhibit the germination of C. albicans in vitro. Germination was induced at $37^{\circ} \mathrm{C}$ in dextrose-free Sabouraud (Joshi et al., 1973), Spider (Liu et al., 1994) or Lee's (Lee et al., 1975) media using the $C$. albicans HWP1$L a c Z$ strain: $\beta$-galactosidase measurements reflect the morphological change from yeast cells to hyphae (Hogan et al., 2004; Clément et al., 2007). The $\beta$-galactosidase measurements were normalized using the XTT assay (Honraet et al., 2005) to correct for biomass increase.

The FFA originating from the saponification of total whey cream lipids are active at inhibiting the germination of C. albicans in all inducing media used, but this activity seems to be stronger in Lee's and Spider media (Figure 1A). We have shown previously that unsaturated FFA from bovine whey exhibits a strong antigermination activity (Clément et al., 2007). Fractionation of our saponified FFA by the urea inclusion procedure, which permits separation of straight-chain (i.e., mainly saturated) from non-straight-chain (i.e., mainly unsaturated and branched-chain) FFA, revealed that the latter was the most active one (Figure 1A). Indeed, whereas unfractionated and straight-chain FFA-enriched fractions inhibited the germination of C. albicans in dextrose-free Sabouraud by about $40 \%$, this inhibition reached about $80 \%$ with the non-straight-chain FFA enriched fraction (Figure 1A). Similar observations were made in Lee's and Spider inducing media, indicating that these effects are not restricted to a specific condition (Figure 1A). Microscopic observations of cells after incubation indicated that cellular morphologies (Figure 1B) correlated with the $\beta$-galactosidase data (Figure 1A). It is noteworthy that the non-straightchain FFA enriched fraction represents about $0.3 \%$ of whey cream.

The results presented above indicate that although FFA fractions are active at inhibiting the germination of C. albicans, they do not compromise its multiplication as a budding yeast. However, it is known that the inoculum can affect the activity of some antimicrobials. This in vitro phenomenon, termed inoculum effect, refers to the MIC of a drug increasing with respect to the inoculum (Brook, 1989; Rex et al., 1993; Stratton, 1993; LassFlorl et al., 2003). The antigermination activity of our FFA fractions was determined using an inoculum of $10^{5} \mathrm{cfu} / \mathrm{mL}$ and a FFA concentration of $10 \mu \mathrm{g} / \mathrm{mL}$. To evaluate whether our FFA fractions could also inhibit the growth of both morphological forms of C. albicans (i.e., blastospores and hyphae), they were tested on a lower inoculum of the fungus (i.e., $10^{3} \mathrm{cfu} / \mathrm{mL}$ ), as suggested (Jacob and Walker, 2005). We were interested in the activity inhibiting the growth of fungi because such an approach allows purification and identification of both fungistatic and fungicidic molecules. Whereas unfractionated and straight-chain FFA fractions can inhibit the germination of $C$. albicans in vitro (Figure 1 ), none of these fractions can interfere with the normal multiplication of this fungus at low inoculum (Figure 2A). However, the non-straight-chain FFA-enriched fraction inhibits, in a dose-dependent manner, the growth of C. albicans (Figure 2A). This antifungal activity was not restricted to this fungus as this same frac- 

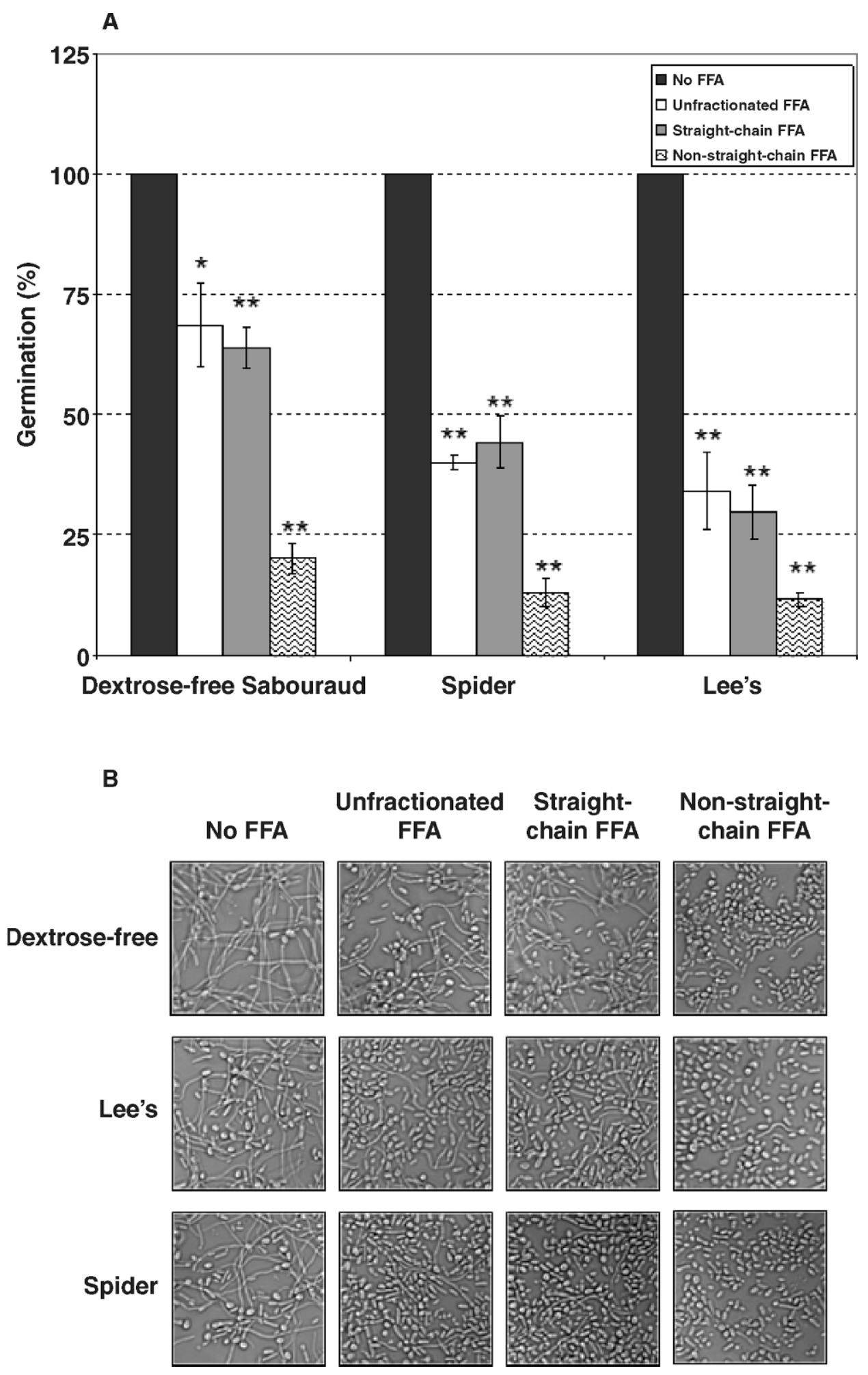

Figure 1. Inhibition of Candida albicans germination in different inducing media by bovine whey chain FFA-enriched fractions. Blastospores of the Candida albicans HWP1-LacZ strain $\left(5 \times 10^{5}\right.$ spores $\left./ \mathrm{mL}\right)$ were induced to germinate at $37^{\circ} \mathrm{C}$ during $4 \mathrm{~h}$ in dextrose-free Sabouraud, Spider, or Lee's media. Each of the FFA fractions was used at a concentration of $10 \mu \mathrm{g} / \mathrm{mL}$. (A) The percentage of germination represents the level of $\beta$-galactosidase expression, which is under the control of the HWP1 promoter expressed in hyphae, of cells incubated with samples to sample free control and corrected for biomass using XTT. *Indicates $P<0.02$ and $* *$ indicates $P<0.005$ when compared with the sample-free control. (B) Microscopic observations of cells after $4 \mathrm{~h}$ of incubation at $37^{\circ} \mathrm{C}$ in different inducing media. 
A
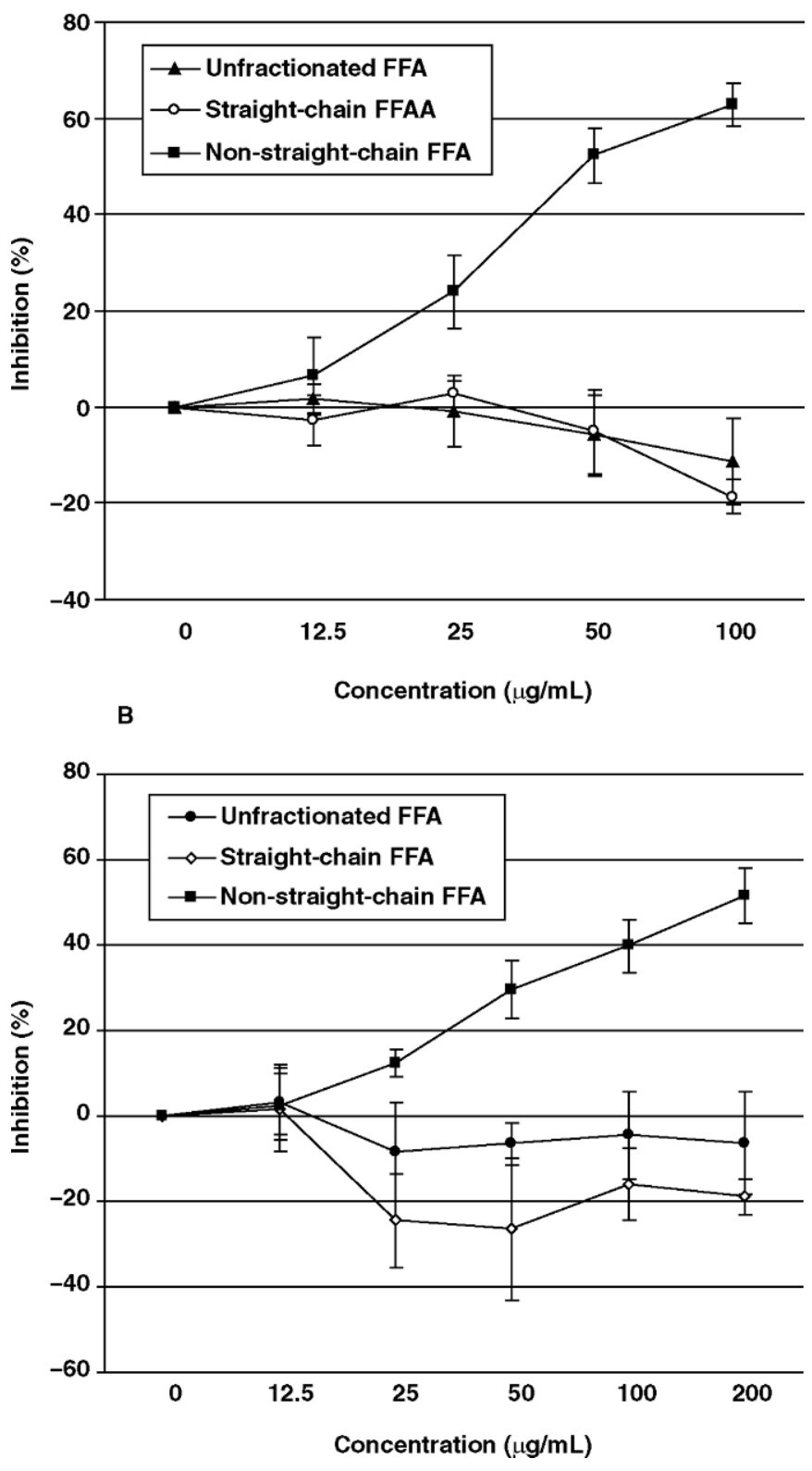

Figure 2. Antifungal activity of bovine non-straight-chain chain FFA-enriched fraction. (A) Blastospores of Candida albicans $\left(5 \times 10^{3}\right.$ spores $/ \mathrm{mL}$ ) incubated at $37^{\circ} \mathrm{C}$ for $48 \mathrm{~h}$ in dextrose-free Sabouraud liquid medium containing FFA, non-straight-chain FFA, or straightchain FFA at the indicated concentrations. (B) Spores of A. fumigatus $\left(5 \times 10^{3}\right.$ spores $\left./ \mathrm{mL}\right)$ were incubated at $37^{\circ} \mathrm{C}$ for $48 \mathrm{~h}$ in Sabouraud liquid medium supplemented with the indicated concentrations of FFA fractions. The growth inhibition (\%) represent the reduction of absorbance at $595 \mathrm{~nm}$ of spores incubated with samples to sample free control. Error bars represent standard deviations for fourth experiments.

tion could also inhibit the growth of A. fumigatus, an emerging fungal pathogen growing exclusively as hyphae (Figure 2B). It is noteworthy to mention that the non-straight-chain FFA enriched fraction did not significantly impair the growth of $C$. albicans at an inoculum of $10^{5} \mathrm{cfu} / \mathrm{mL}$, at least at a concentration of $50 \mu \mathrm{g} /$ $\mathrm{mL}$ (data not shown). These observations indicate that non-straight-chain FFA from bovine whey can inhibit the germination and growth of 2 human fungal pathogens and that these in vitro activities are affected by the inoculum size. This suggests that at a high inoculum, the number of targets or molecules mediating this phenomenon may exceed the capacity of our active FFA to inhibit the growth of C. albicans. Similar observations were reported using some azole antifungals, which inhibit the germination of $C$. albicans at subinhibitory concentrations (Johnson et al., 1983, 1984; Odds et al., 1985).

\section{Isolation and Identification of Antifungal Compounds}

The GC analysis revealed that the composition of the non-straight-chain FFA fraction was highly complex and enriched in at least 17 different FFA, including capric, myristoleic, linoleic, and arachidonic acids (Table 1). Because these FFA were previously identified as active compounds inhibiting the germination of $C$. albicans in vitro (Clément et al., 2007), it is tempting to suggest that they could account for the higher activity of the non-straight-chain FFA-enriched fraction. However, this fraction also contains other FFA that have not yet been shown as having an activity against the germination of C. albicans. On the other hand, GC analysis of the straight-chain FFA enriched fractions indicated that it was fairly similar in composition to unfractionated FFA and still contained unsaturated FFA (Table 1). This suggests that only a small fraction of bovine unsaturated FFA, branched-chain FFA, or both had been separated from straight-chain FFA by the urea inclusion procedure. Nevertheless, this similarity in composition is consistent with the finding that both fractions inhibit the germination of $C$. albicans at similar levels in every inducing medium used (Figure 1).

The ability of the non-straight-chain FFA enriched fraction to inhibit the growth of both fungal pathogens was further investigated using an antifungal assayguided HPLC fractionation. Using this procedure, 5 fractions (F7, F8, F11, F19, and F20) exhibiting an antifungal activity against both $A$. fumigatus and $C$. albicans were identified. In addition, 2 other fractions (F22 and F30) were found to be active only against $C$. albicans. The HPLC chromatogram of the non-straightchain FFA enriched fraction is shown in Figure 3. Methyl ester derivatives of components present in active fractions were analyzed using capillary gas chromatography. According to a reference FAME mixture, 
Table 1. Composition (\%) of total chain FFA and its 2 fractions

\begin{tabular}{|c|c|c|c|}
\hline Identity $^{1}$ & FFA & Straight-chain & Non-straight-chain \\
\hline C8:0 & 1.46 & $-^{2}$ & 0.76 \\
\hline C10:0 & 2.02 & 0.56 & 18.4 \\
\hline$?$ & 0.092 & - & 1.31 \\
\hline C11:0 & - & - & 0.25 \\
\hline C12:0 & 4.02 & 3.04 & 5.04 \\
\hline$?$ & - & - & 1.1 \\
\hline C12:1? & 0.31 & - & 1.05 \\
\hline$?$ & - & - & 0.6 \\
\hline$?$ & - & - & 0.28 \\
\hline C13:0 & 0.12 & 0.098 & $14^{*}$ \\
\hline$?$ & 0.14 & 0.1 & 1.4 \\
\hline C14:0 & 16.2 & 14.59 & 0.32 \\
\hline $\mathrm{C} 14: \ln 5$ & 0.94 & 0.72 & 13.6 \\
\hline$?$ & 0.28 & 0.26 & 0.46 \\
\hline$?$ & 0.06 & 0.43 & - \\
\hline C15:0 & 1.56 & 1.44 & $8.37^{*}$ \\
\hline C15:1 & 0.44 & 0.41 & 0.35 \\
\hline C16:0 & 39.6 & 38.7 & 1 \\
\hline $\mathrm{C} 16: \ln 7$ & 1.2 & 1.51 & 1.22 \\
\hline$?$ & 0.18 & 0.19 & 0.3 \\
\hline$?$ & 0.44 & 0.45 & - \\
\hline$?$ & 0.57 & 0.55 & 0.72 \\
\hline $\mathrm{C} 17: 0$ & 0.74 & 0.71 & $0.59 *$ \\
\hline C17:1 & 0.14 & 0.22 & 0.89 \\
\hline C18:0 & 12.06 & 11.21 & 0.74 \\
\hline $\mathrm{C} 18: 1 \mathrm{n} 9 \mathrm{c}$ & 12.8 & 17.4 & 0.83 \\
\hline$?$ & 0.95 & 1.66 & - \\
\hline$?$ & - & 1.01 & - \\
\hline$?$ & 0.15 & 0.15 & - \\
\hline $\mathrm{C} 18: 1 \mathrm{n} 9 \mathrm{t} / \mathrm{C} 18: 2 \mathrm{n} 6 \mathrm{c}$ & 0.86 & 1.21 & 9.8 \\
\hline $\mathrm{C} 18: 2 \mathrm{n} 6 \mathrm{t}$ & 0.29 & 0.29 & 4.9 \\
\hline $\mathrm{C} 18: 3 \mathrm{n} 6$ & - & - & 0.81 \\
\hline C18:3n3 & 0.29 & 0.33 & 3.7 \\
\hline$?$ & - & 0.38 & - \\
\hline C20:3n6 & - & - & 1.46 \\
\hline $\mathrm{C} 20: 4 \mathrm{n} 6$ & - & - & 1.83 \\
\hline
\end{tabular}

${ }^{1}$ Elution order.

${ }^{2}$ Not detected.

*May contain branched-chain FFA.

the antifungal components of each fraction were identified as capric acid (C10:0; F7 and F8), lauroleic acid (C12:1; F11), myristoleic acid (C14:1n-5; F19 and F20), 11-methyldodecanoic acid (iso-C13:0; F22), and $\gamma$-linolenic acid (C18:3n-6; F30). The identity of these FFA was further confirmed using mass spectroscopy and HPLC analysis with commercial reference compounds when they were available (data not shown).

To confirm the ability of these FFA to inhibit the growth of fungal pathogens, we carried out quantitative assays using commercial preparations (Table 2). These assays were done using dextrose-free Sabouraud $(C$. albicans) or Sabouraud dextrose (A. fumigatus) because these media were used during our assay-guided HPLC fractionation. Lauroleic acid was excluded from our assays because the isomer present in bovine milk (C12:1n-3) was not commercially available. As expected, all of the identified FFA exhibited an in vitro antifungal activity, with $C$. albicans being generally more susceptible than A. fumigatus, except for capric acid (Table 2, Sabouraud column). Capric and myristoleic acids inhibited by $50 \%$ the growth of $A$. fumigatus at 20 and $34 \mathrm{mg} / \mathrm{L}$, respectively. These 2 FFA were also active against $C$. albicans with $\mathrm{MIC}_{50}$ of 30 and $16 \mathrm{mg} /$ $\mathrm{L}$, respectively. The $\gamma$-linolenic acid, which was initially isolated based on its ability to inhibit the growth of $C$. albicans, was found to be active against both C. albicans and $A$. fumigatus in vitro. Interestingly, $\gamma$-linolenic acid was the FFA exhibiting the highest antifungal activity with $\mathrm{MIC}_{50}$ of 0.66 and $1.2 \mathrm{mg} / \mathrm{L}$ against $C$. albicans and A. fumigatus, respectively. Similarly, 11-methyldodecanoic acid was active against $C$. albicans and $A$. fumigatus, even if it was only isolated based on its ability to inhibit the growth of $C$. albicans. As shown in Table 2, about 8.4 and $100 \mathrm{mg} / \mathrm{L}$ of 11 -methyldodecanoic acid were required to inhibit $50 \%$ of $C$. albicans and A. fumigatus growth, respectively. Previously, capric, myristoleic, linoleic, and arachidonic acids were found to exhibit antigermination activities against $C$. albicans in vitro (Clément et al., 2007). The finding in the present study of identical (i.e., capric and myristoleic acids) and different (i.e., lauroleic, 11-méthyldodecanoicm, and $\gamma$ linolenic acids) FFA inhibiting the growth of both morphological forms of C. albicans suggests that there is no clear correlation between the antigermination and the growth inhibition activities of FFA.

As medium composition and $\mathrm{pH}$ are known to affect the activity of some antifungal drugs, ability of the identified FFA to inhibit the growth of fungal pathogens was also determined in RPMI-1640 cell culture medium buffered with MOPS at $\mathrm{pH}$ 7.0, as recommended (Jacob and Walker, 2005). These results indicate that most of the identified FFA are active at inhibiting the growth of C. albicans and A. fumigatus in this incubating medium (Table 2, RPMI-1640 column). In contrast to the data obtained in Sabouraud media, C. albicans seemed to be generally less susceptible than A. fumigatus in RPMI$1640 \mathrm{pH} 7$ (Table 2), but the highest antifungal activity was still observed with $\gamma$-linolenic acid. Indeed, the concentration of this FFA required for 50\% inhibition of the growth of C. albicans and A. fumigatus was 5.4 and $1.3 \mathrm{mg} / \mathrm{L}$, respectively (Table 2 ). The 11-methyldodecanoic and myristoleic acids also exhibited antifungal activities against both $C$. albicans $\left(\mathrm{MIC}_{50}\right.$ of 30 and 26 $\mathrm{mg} / \mathrm{L}$, respectively) and A. fumigatus $\left(\mathrm{MIC}_{50}\right.$ of 9.5 and $6.5 \mathrm{mg} / \mathrm{L}$, respectively). Finally, capric acid was not found to be active against $C$. albicans in RPMI MOPS medium (i.e., $\mathrm{MIC}_{50}$ over $172 \mathrm{mg} / \mathrm{L}$ ), but about $83 \mathrm{mg} /$ $\mathrm{L}$ of this FFA was required to inhibit by $50 \%$ the growth of $A$. fumigatus (Table 2). As a reference compound, 5fluorocytosine exhibited a high antifungal activity against $C$. albicans in RPMI MOPS $\mathrm{pH} 7$ with a $\mathrm{MIC}_{50}$ of $0.21 \mathrm{mg} / \mathrm{L}$ (Table 2). At this time, we cannot explain 


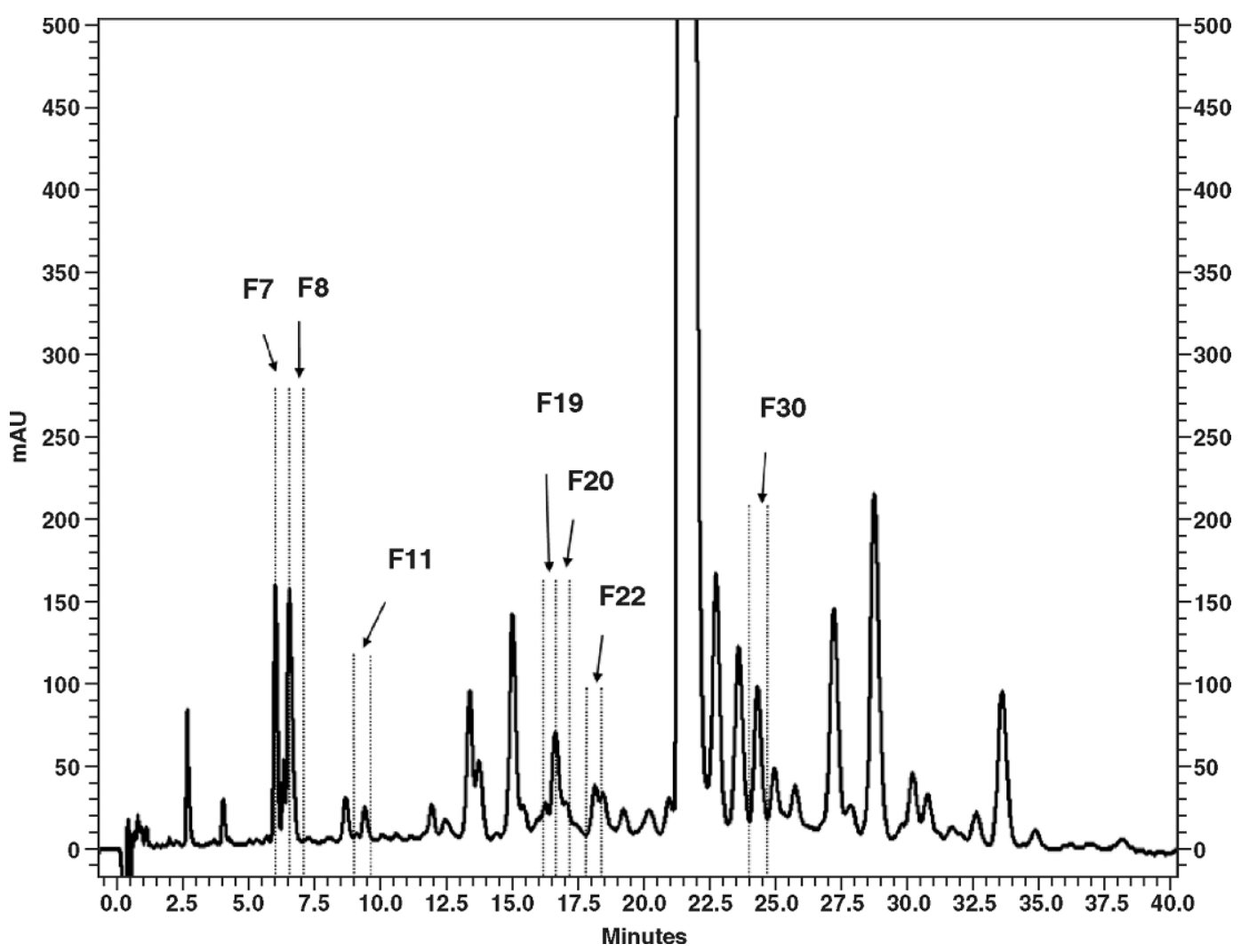

Figure 3. Separation of the non-straight-chain FFA enriched fraction using a reversed-phase Nova-Pak HR C18 semi-prep HPLC column $(7.8 \times 300 \mathrm{~mm}, 6 \mu \mathrm{m})$. Non-straight-chain FFA dissolved in 50\% ethanol was injected manually onto the column and compounds eluting with an increasing concentration of acetonitrile were detected at $215 \mathrm{~nm}$ (milli-arbitrary units, mAU). The flow rate was $8 \mathrm{~mL} / \mathrm{min}$, and each detected peak was manually collected. Fractions exhibiting antifungal activity are indicated.

the medium-dependant levels of antifungal activity of some FFA. However, this phenomenon could involve a faster assimilation of FFA by cells in the dextrose-free Sabouraud medium. Indeed, this medium contains only peptone as a carbon source, compared with RPMI-1640, which contains glucose. In addition, the presence of peptone in dextrose-free medium could also affect the solubility/availability of some FFA by acting as a carrier.

The mechanism by which selected FFA can inhibit the growth of fungal pathogens is currently unknown. Previous studies have demonstrated that medium chain fatty acids such as capric and lauric acids can kill $C$. albicans cells by disrupting/disorganizing the plasma membrane (Bergsson et al., 2001). Medium chain fatty acids have also been shown to increase the rate of passive $\mathrm{H}^{+}$influx across the plasma membrane (Stevens and Hofemyer, 1993), which could interfere with the cytoplasmic alkalinisation that normally accompanies the germination of $C$. albicans (Stewart et al., 1988). Others studies have reported an important contribution of lipids during the germination of C. albicans because (1) cerulenin, an inhibitor of fatty acid biosynthesis (Omura, 1976), can inhibit the germina-

Table 2. Antifungal activities of chain FFA and 5-fluorocytosine (5-FC)

\begin{tabular}{lccccc}
\hline & \multicolumn{2}{c}{$\mathrm{MIC}_{50}(\mathrm{mg} / \mathrm{L})$ Sabouraud } & & \multicolumn{2}{c}{ RPMI-1640 at $\mathrm{pH} 7.0(\mathrm{MOPS})$} \\
\cline { 2 - 3 } \cline { 5 - 6 } Compound & Aspergillus fumigatus & Candida albicans & & A. fumigatus & \multicolumn{2}{c}{ C. albicans } \\
\hline Capric acid (C10:0) & $20(14-24)$ & $30(27-32)$ & & $83(49-106)$ & $>1,000$ \\
Myristoleic acid (C14:1n-5) & $34(18-50)$ & $16(15-17)$ & & $6.5(2.4-10.3)$ & $26(22-31)$ \\
11-Methyldodecanoic acid (C13:0i) & $100(69-143)$ & $8.4(7.2-9.6)$ & & $9.5(6.3-11.6)$ & $30(18-36)$ \\
$\gamma$-Linolenic acid (C18:3n-6) & $1.2(0.9-1.4)$ & $0.66(0.61-0.74)$ & & $1.3(0.6-2.2)$ & $5.4(3-6.8)$ \\
5-FC & ND & ND & & ND & $0.21(0.12-0.26)$ \\
\hline
\end{tabular}


tion of C. albicans (Hoberg et al., 1983); (2) a C. albicans phospholipase D1 null mutant fails to form hyphae (Dolan et al., 2004); (3) a modest reduction in oleic acid in $C$. albicans was found to prevent the formation of hyphae (Krishnamurthy et al., 2004); and (4) pathogenic fungi were recently found to produce secondary metabolites derived from the oxidation of fatty acids (Tsitsigiannis and Keller, 2007). These molecules, named oxylipins, include farnesol, a quorum-sensing molecule that inhibits the germination of C. albicans (Hornby et al., 2001), and eicosanoids, which can alter fungal development (Noverr et al., 2002). Whether our whey-derived fatty acids could inhibit the growth of both fungal pathogens by one of these pathways requires further investigation. However, only capric, lauroleic, 11-methyldodecanoic, and $\gamma$-linolenic were isolated and identified in our in vitro assay-guided fractionation. Other fractions containing abundant unsaturated milk fatty acids such as $\alpha$-linolenic and linoleic acids were not found to be active. Therefore, this suggests that the growth inhibition observed by specific FFA is unlikely to be the consequence of a nonspecific mechanism related to the presence of any FFA in the incubating medium, but rather to specific structural determinants present in these active molecules.

\section{CONCLUSIONS}

Germination is believed to be important for the virulence of $C$. albicans, particularly as mutants that fail to form hyphae exhibit reduced virulence in animal models. In addition, genes encoding virulence factors such as adhesins and proteases are preferentially expressed during filamentation (Kumamoto and Vinces, 2005). Targeting yeast to hyphae transition instead of focusing on compounds exhibiting fungicidal/static activity was indeed suggested as a novel approach in antifungal drug discovery, which is believed to be less selective for drug-resistant strains (Jiang et al., 2002). The finding of bovine whey FFA blocking the germination of C. albicans follows this new trend and could eventually lead to the development of new antifungal agents/therapies. In addition to this activity, some bovine whey FFA were found to inhibit growth of both developmental forms of $C$. albicans as well as A. fumigatus. As this activity was found to be affected by the inoculum, this suggests that such molecules could be inefficient as a therapeutic agent to cure established fungal infections (i.e., high inoculum). On the other hand, they could alone or in combination with other milk/whey components exhibit activity in the prevention of fungal infections (i.e., low inoculum), which could confer an interesting prophylactic activity to bovine milk/whey components. Finally, the finding that semi-purified FFA- enriched fractions derived from bovine whey can also inhibit the germination and the growth of fungi is attractive, particularly because the methods used to obtain them are industrially compatible.

\section{ACKNOWLEDGMENTS}

We are grateful to Sebastien Sauvé, Pedro A. Segura, and Paul B. Fayad for GC analysis (Université de Montréal, Montréal); Orval Mamer, and Alain Lesimple for mass spectroscopy analysis (McGill University, Montréal); and Jeanine Joly for the A. fumigatus strain (Université de Montréal, Montréal). We would like to thank Michel Lafleur, Julie Shareck, and Sébastien Croisetière for helpful discussions and Saputo (Montréal, Canada) for excellent collaboration and support. We would finally like to thank Julie Shareck and Patrick $\mathrm{C}$. Hallenbeck for critical reading. $\mathrm{PB}$ is the recipient of the Saputo Research Chair in Biomedical Dairy Products Optimization of Université de Montréal. This work was supported by NSERC/Dairy Farmers of Canada (CRD Program).

\section{REFERENCES}

Al Jasser, A. M., and N. A. Elkhizzi. 2004. Distribution of Candida species among bloodstream isolates. Saudi Med. J. 25:566-569.

Bergsson, G., J. Arnfinnsson, O. Steingrimsson, and H. Thormar. 2001. In vitro killing of Candida albicans by fatty acids and monoglycerides. Antimicrob. Agents Chemother. 45:3209-3212.

Bligh, E. G., and W. J. Dyer. 1959. A rapid method of total lipid extraction and purification. Can. J. Med. Sci. 37:911-917.

Brook, I. 1989. Inoculum effect. Rev. Infect. Dis. 11:361-368.

Calderone, R. A., and W. A. Fonzi. 2001. Virulence factors of Candida albicans. Trends Microbiol. 9:327-335.

Cimon, B., J. Carrere, J. F. Vinatier, J. P. Chazalette, D. Chabasse, and J. P. Bouchara. 2000. Clinical significance of Scedosporium apiospermum in patients with cystic fibrosis. Eur. J. Clin. Microbiol. Infect. Dis. 19:53-56.

Clark, T. A., and R. A. Hajjeh. 2002. Recent trends in the epidemiology of invasive mycoses. Curr. Opin. Infect. Dis. 15:569-574.

Clément, M., J. Tremblay, M. Lange, J. Thibodeau, and P. Belhumeur. 2007. Whey-derived free fatty acids suppress the germination of Candida albicans in vitro. FEMS Yeast Res. 7:276-285.

Denning, D. W. 1998. Invasive aspergillosis. Clin. Infect. Dis. 26:781-803.

Dolan, J. W., A. C. Bell, B. Hube, M. Schaller, T. F. Warner, and E. Balish. 2004. Candida albicans PLD I activity is required for full virulence. Med. Mycol. 42:439-447.

Fonzi, W. A., and M. Y. Irwin. 1993. Isogenic strain construction and gene mapping in Candida albicans. Genetics 134:717-728.

Groll, A. H., and T. J. Walsh. 2001. Uncommon opportunistic fungi: New nosocomial threats. Clin. Microbiol. Infect. 7(Suppl. 2):8-24.

Hajjeh, R. A., A. N. Sofair, L. H. Harrison, G. M. Lyon, B. A. Arthington-Skaggs, S. A. Mirza, M. Phelan, J. Morgan, W. Lee-Yang, M. A. Ciblak, L. E. Benjamin, L. T. Sanza, S. Huie, S. F. Yeo, M. E. Brandt, and D. W. Warnock. 2004. Incidence of bloodstream infections due to Candida species and in vitro susceptibilities of isolates collected from 1998 to 2000 in a population-based active surveillance program. J. Clin. Microbiol. 42:1519-1527.

Hoberg, K.A., R.L. Cihlar, and R.A. Calderone. 1983. Inhibitory effect of cerulenin and sodium butyrate on germination of Candida albicans. Antimicrob. Agents Chemother. 24:401-408. 
Hogan, D. A., A. Vik, and R. Kolter. 2004. A Pseudomonas aeruginosa quorum-sensing molecule influences Candida albicans morphology. Mol. Microbiol. 54:1212-1223.

Honraet, K., E. Goetghebeur, and H. J. Nelis. 2005. Comparison of three assays for the quantification of Candida biomass in suspension and CDC reactor grown biofilms. J. Microbiol. Methods 63:287-295.

Hornby, J. M., E. C. Jensen, A. D. Lisec, J. J. Tastos, B. Jahnke, R. Shoemaker, P. Dussault, and K. W. Nickerson. 2001. Quorum sensing in the dimorphic fungus Candida albicans is mediated by farnesol. Appl. Environ. Microbiol. 67:2982-2992.

Huth, P. J., D. B. DiRienzo, and G. D. Miller. 2006. Major scientific advances with dairy foods in nutrition and health. J. Dairy Sci. 89:1207-1221.

Idigoras, P., E. Perez-Trallero, L. Pineiro, J. Larruskain, M. C. LopezLopategui, N. Rodriguez, and J. M. Gonzalez. 2001. Disseminated infection and colonization by Scedosporium prolificans: A review of 18 cases, 1990-1999. Clin. Infect. Dis. 32:E158-E165.

Isaacs, C. E. 2001. The antimicrobial function of milk lipids. Adv. Nutr. Res. 10:271-285.

Jackson, B. E., K. R. Wilhelmus, and B. M. Mitchell. 2007. Genetically regulated filamentation contributes to Candida albicans virulence during corneal infection. Microb. Pathog. 42:88-93.

Jacob, M. R., and L. A. Walker. 2005. Natural products and antifungal drug discovery. Methods Mol. Med. 118:83-109.

Jensen, R. G. 2002. The composition of bovine milk lipids: January 1995 to December 2000. J. Dairy Sci. 85:295-350.

Jiang, B., H. Bussey, and T. Roemer. 2002. Novel strategies in antifungal lead discovery. Curr. Opin. Microbiol. 5:466-471.

Johnson, E. M., M. D. Richardson, and D. W. Warnock. 1983. Effect of imidazole antifungals on the development of germ tubes by strains of Candida albicans. J. Antimicrob. Chemother. 12:303-316.

Johnson, E. M., M. D. Richardson, and D. W. Warnock. 1984. Invitro resistance to imidazole antifungals in Candida albicans. J. Antimicrob. Chemother. 13:547-558.

Joshi, K. R., J. B. Gavin, and D. A. Bremner. 1973. The formation of germ tubes by Candida albicans in various peptone media. Sabouraudia 11:259-262.

Kabara, J. J., D. M. Swieczkowski, A. J. Conley, and J. P. Truant. 1972. Fatty acids and derivatives as antimicrobial agents. Antimicrob. Agents Chemother. 2:23-28.

Kaluzny, M. A., L. A. Duncan, M. V. Merritt, and D. E. Epps. 1985. Rapid separation of lipid classes in high yield and purity using bonded phase columns. J. Lipid Res. 26:135-140.

Kippert, F. 1995. A rapid permeabilization procedure for accurate quantitative determination of beta-galactosidase activity in yeast cells. FEMS Microbiol. Lett. 128:201-206.

Kontoyiannis, D. P., and R. E. Lewis. 2002. Antifungal drug resistance of pathogenic fungi. Lancet 359:1135-1144.

Krishnamurthy, S., A. Plaine, J. Albert, T. Prasad, R. Prasad, and J. F. Ernst. 2004. Dosage-dependent functions of fatty acid desaturase Ole1p in growth and morphogenesis of Candida albicans. Microbiology 150:1991-2003.

Kullberg, B. J., and A. M. Oude Lashof. 2002. Epidemiology of opportunistic invasive mycoses. Eur. J. Med. Res. 7:183-191.

Kumamoto, C. A., and M. D. Vinces. 2005. Contributions of hyphae and hypha-co-regulated genes to Candida albicans virulence. Cell. Microbiol. 7:1546-1554.

Lass-Florl, C., C. Speth, G. Kofler, M. P. Dierch, E. Gunsilius, and R. Wurzner. 2003. Effect of increasing inoculum sizes of Aspergillus hyphae on MICs and MFCs of antifungal agents by broth microdilution method. Int. J. Antimicrob. Agents 21:229-233.

Latge, J. P. 1999. Aspergillus fumigatus and aspergillosis. Clin. Microbiol. Rev. 12:310-350.
Lee, K. L., H. R. Buckley, and C. C. Campbell. 1975. An amino acid liquid synthetic medium for the development of mycelial and yeast forms of Candida albicans. Sabouraudia 13:148-153.

Liu, H., J. Kohler, and G. R. Fink. 1994. Suppression of hyphal formation in Candida albicans by mutation of a STE12 homolog. Science 266:1723-1726.

Lo, H. J., J. R. Kohler, B. DiDomenico, D. Loebenberg, A. Cacciapuoti, and G. R. Fink. 1997. Nonfilamentous C. albicans mutants are avirulent. Cell 90:939-949.

Molkentin, J. 1999. Bioactive lipids naturally occurring in bovine milk. Nahrung 43:185-189.

Noverr, M. C., G. B. Toews, and G. B. Huffnagle. 2002. Production of prostaglandins and leukotrienes by pathogenic fungi. Infect. Immun. 70:400-402.

Odds, F. C., A. Cockayne, J. Hayward, and A. B. Abbott. 1985. Effects of imidazole- and triazole-derivative antifungal compounds on the growth and morphological development of Candida albicans hyphae. J. Gen. Microbiol. 131:2581-2589.

Omura, S. 1976. The antibiotic cerulenin, a novel tool for biochemistry as an inhibitor of fatty acid synthesis. Bacteriol. Rev. 40:681-697.

Patterson, T. F. 2001. Invasive mycoses: management and unmet medical needs. Curr. Opin. Infect. Dis. 14:669-671.

Redding, S. W. 2001. The role of yeasts other than Candida albicans in oropharyngeal candidiasis. Curr. Opin. Infect. Dis. 14:673-677.

Rex, J. H., M. A. Pfaller, M. G. Rinaldi, A. Polak, and J. N. Galgiani. 1993. Antifungal susceptibility testing. Clin. Microbiol. Rev. 6:367-381.

Sanglard, D., and F. C. Odds. 2002. Resistance of Candida species to antifungal agents: Molecular mechanisms and clinical consequences. Lancet Infect. Dis. 2:73-85.

Saville, S. P., A. L. Lazzell, A. P. Bryant, A. Fretzen, A. Monreal, E. O. Solberg, C. Monteagudo, J. L. Lopez-Ribot, and G. T. Milne. 2006. Inhibition of filamentation can be used to treat disseminated candidiasis. Antimicrob. Agents Chemother. 50:3312-3316.

Severin, S., and X. Wenshui. 2005. Milk biologically active components as nutraceuticals. Crit. Rev. Food Sci. Nutr. 45:645-656. (Review).

Shah, N. P. 2000. Effects of milk-derived bioactives: An overview. Br. J. Nutr. 84(Suppl. 1):S3-S10.

Sprong, R. C., M. F. Hulstein, and M. R. Van der Meer. 2001. Bactericidal activities of milk lipids. Antimicrob. Agents Chemother. 45:1298-1301.

Stevens, S., and J.-H. S. Hofemyer. 1993. Effects of ethanol, octanoic and decanoic acids on fermentation and the passive influx of protons through the plasma membrane of Saccharomyces cerevisiae. Appl. Microbiol. Biotechnol. 38:656-663.

Stewart, E., N. A. Gow, and D. V. Bowen. 1988. Cytoplasmic alkalinisation during germ tube formation in Candida albicans. J. Gen. Microbiol. 134:1079-1087.

Stratton, C. W. 1993. Bactericidal testing. Infect. Dis. Clin. North Am. 7:445-459.

Tortorano, A. M., L. Caspani, A. L. Rigoni, E. Biraghi, A. Sicignano, and M. A. Viviani. 2004. Candidosis in the intensive care unit: A 20-year survey. J. Hosp. Infect. 57:8-13.

Traitler, H., H. J. Wille, and A. Studer. 1988. Fractionation of blackcurrant seed oil. J. Am. Oil Chem. Soc. 65:755-760.

Tsitsigiannis, D. I., and N. P. Keller. 2007. Oxylipins as developmental and host-fungal communication signals. Trends Microbiol. 15:109-118.

Vaghela, M. N., and A. Kilara. 1995. A rapid method for extraction of total lipids from whey-protein concentrates and separation of lipid classes with solid-phase extraction. J. Am. Oil Chem. Soc. 72:1117-1121.

Walzem, R. L., C. J. Dillard, and J. B. German. 2002. Whey components: Millennia of evolution create functionalities for mammalian nutrition: what we know and what we may be overlooking. Crit. Rev. Food Sci. Nutr. 42:353-375. 